\title{
Editorial
}

\section{Antimicrobial Resistance- a Global Concern for Human Survival}

\section{F Ahammad}

Antimicrobial resistance (AMR) is a form of drug resistance whereby some (or, less commonly, all) subpopulations of a microorganism, usually a bacterial species, are able to survive after exposure to one or more antibiotics. Pathogens resistant to multiple antibiotics are considered multidrug resistant (MDR) superbugs ${ }^{1}$. The modern era of antimicrobial chemotherapy starts in 1936 with the introduction of sulfanilamide into clinical practice. Penicillin becomes available in quantities sufficient for clinical use in $1941^{2}$. Streptomycin, chloramphenicol, and chlortetracycline were identified at the end of or soon after World War II. Since then development of AMR and invention of new classes of antimicrobials to overcome the situation are simultaneously going on. Carbapenems the most powerful beta lactamase inhibitors already have been shown resistance. To combat the situation there is no noble antibiotic in pipeline and scientists are not hopeful to get a new antibiotic up to 2025. So the world is passing the hopeless, frustrating era.

Mechanism of AMR: Resistance may be acquired by mutation and selection, with passage of the trait vertically to daughter cells. More commonly, resistance is acquired by horizontal transfer of determinants from a donor cell, often of another bacterial species, by transduction, transformation, or conjugation. Horizontal transfer can become rapidly and widely disseminated either by clonal spread of the resistant strain itself or by further genetic transfers from resistant strain to other susceptible strains. AMR falls into three general categories: (1) the drug can't reach its target due to failure to cross the cell membrane of the microbes; (2) inactivation of the drug by production of enzymes like beta- lactamase,; and (3) alteration of target by mutation of the natural target, target modification, or substitution with a resistant alternative to the native ${ }^{2}$.

Ways of AMR: Both medical professional and animal farmers are contributing to creat the problem. Medical professional are responsible by injudicious use of antibiotic; viral infections are not stopped by antibiotics, yet doctors still prescribe antibiotics to treat them. They are also responsible for under dosing / overdosing, wrong selection of the drug due to poor

Address of correspondence :

Dr. Faruk Ahammad, FCPS. Assoc. Prof. of medicine. Faridpur medical college. Faridpur. Cell no. +8801817011894.

Email-farukahammad26@yahoo.com knowledge about the choice of antibiotic, and also by selling the drugs over the counter without prescription of registered physicians. Antibiotics are used for disease prevention and maximum growth of food producing animals by veterinary and poultry farmers. Use of antibiotics for agricultural purposes, particularly for growth enhancement, has come under much scrutiny, as it has been shown to contribute to the increased prevalence of antibiotic-resistant bacteria of human significance ${ }^{3}$.

Only after 3 years of widely use of penicillin ,Staph. aureus developed penicillin resistance by producing penicillinase enzymes. Methicillin in to use in 1960, unfortunately just after one year in 1961 methicillin rasistance Staph.aureus (MRSA) developed. In these ways so many organisms developed the resistant strains to survive in the world in the battle with antibiotics such as PISP (penicillin intermediate Strept. Pneu.) in 1967, penicillinase-producing H.influenzae in 1974, PRSP (penicillin resistant strept. Pneu.) in 1977, BLNAR (beta lactamase negative ampicillin Resistant) H.influenzae in 1980, ESBL (extended spectrum beta lactamase)-producing Gram-negative bacilli in 1983, VRE (vancomycin resistant E. coli) in 1986 and increase of quinolone-resistant E.coli since 2004. Lastly In the United States and United Kingdom, strains of carbapenem-resistant enteric bacteria have been isolated from patients having received recent medical care in Pakistan, Bangladesh, and India. These strains carry a gene called New Delhi metallo- $\beta$ lactamase (shortened NDM-1) that is responsible for the production of a metallo- $\beta$-lactamase enzyme that hydrolyses carbapenem ${ }^{4}$. A clinical isolate of E. coli from the sputum sample of a patient admitted to a Beijing hospital was found to show unusual resistance to carbapenem that does not rely on the presence of carbapenemase rather by four separate mutations to acquire the resistance to carbapenems ${ }^{5}$. In 2014, researchers in Canada found carbapenem-resistant bacteria in a sample of frozen squid sold at a Chinese grocery store in Saskatoon, Canada. The squid reportedly originated in South Korea and was purchased in January $2014^{6}$.

A World Health Organization report released April 30, 2014 states, "this serious threat is no longer a prediction for the future, it is happening right now in 
every region of the world and has the potential to affect anyone, of any age, in any country. Antibiotic resistance-when bacteria change so antibiotics no longer work in people who need them to treat infections-is now a major threat to public health ${ }^{7}$.

As a response of global concern and crisis European union (EU) has submitted an action plan to Europian parliament with following recommendations- ${ }^{8}$

* Strengthen national multisectorial co-ordination for the containment of antibiotic resistance

* Strengthen surveillance of antibiotic resistance

* Promote strategies for the rational use of antibiotics and strengthen surveillance of antibiotic consumption

* Strengthen infection control and surveillance of antibiotic resistance in health care settings

* Prevent and control the development and spread of antibiotic resistance in the veterinary and agricultural sectors

* Promote innovation and research on new drugs and technology

* Improve awareness, patient safety and partnership

* Considerations for implementation and monitoring

The U.S. Food and Drug Administration has taken written consent from all but one animal drug company for withdrawal of approvals for any production uses of affected drug applications and change the remaining therapeutic uses of their products from over-the-counter (OTC) to use by Veterinary Feed Directive (VFD) or prescription.9 As a part of global village we should take some actions to overcome this crisis. In this regard Clinicians hold the solution and they can play the key roles to face this problem. Following recommendations may be suggested to them to follow-

* Vaccinate population against possible infectious diseases.

* Get the catheters out as early as possible.

* Use local data, or guideline to use antimicrobials, which has made on the basis of local sensitivity.

* Treat infection, not contamination or colonization.

* Isolate the pathogen

*Stop treatment when cured

* Avoid overdosing or under dosing.

* Access the experts when necessary

In conclusion antibiotic resistance is a serious and growing phenomenon in contemporary medicine and has emerged as one of the pre-eminent public health concerns of the 21 st century. There is no noble effective antimicrobial in the pipeline, for which we may wait even up to 2025 . So this is the high time to be rational to use the antimicrobials and to keep in mind that, for the bad bugs; there is no drug.

\section{References :}

1. "Antibiotic Resistance Questions \& Answers". Get Smart: Know When Antibiotics Work. Centers for Disease Control and Prevention, USA. 30 June 2009. [Updated 20 March 2013]. Available from: http://www.cdc.gov/getsmart/antibioticuse/antibiotic-resistance-faqs.html\#e

2. Henry F. Chambers. Antimicrobial agents. In: Joel G.Hardman, Lee E. Limbird, editors. Goodman \& Gilman's The pharmacological basis of therapeutics. 10th ed. International edition: McGraw-Hill; 2001: p 1143-45.

3. Mathew $\mathrm{AG}^{1}$, Cissell $\mathrm{R}$, Liamthong $\mathrm{S}$. Antibiotic resistance in bacteria associated with food animals: a United States perspective of livestock production. Foodborne Pathog Dis. 2007 Summer;4(2):115-33.

4. "Detection of Enterobacteriaceae isolates carrying metallo-betalactamase - United States, 2010". Morbidity and Mortality Weekly Report 59 (24): 750 . PMID 20577157.

5. Levy Stuart, Warner Douglas, Yang Qiwen, Duval Valerie, Chen Minjun, Xu Yingchun, et al. Involvement of MarR and YedS in Carbapenem Resistance in a Clinical Isolate of Escherichia coli from China. Antimicrobial Agents and Chemotherapy 2013;57 (4): 1935-1937.

6. Lena H. Sun. "Bacteria found in squid raises concern about spread of antibiotic resistance, study finds". Washington Post. June 11, 2014.

7. WHO. Antimicrobial resistance: global report on surveillance 2014. April 2014 (Number of pages: 257).ISBN: 9789241564748.

8. European commission.Road map against antimicrobial resistance. Public health. 2011.Nov.15.

9. U.S. Food and Drug Administration. Animal \& Veterinary. FDA Receives Strong Industry Commitment for its Antibiotic Resistance Strategy. March 26, 2014. 